\title{
Asymptomatic carotid artery stenosis (ACAS)
}

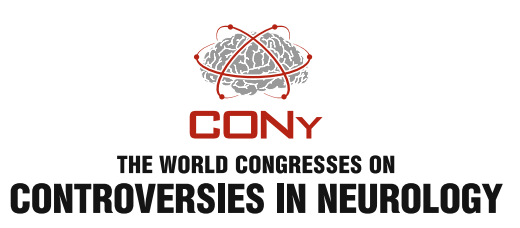

(C) Springer-Verlag 2011

For decades asymptomatic carotid artery stenosis (ACAS) has been a matter of debate. Based on a simplistic view, all stenosed vessels should be cleaned, and the earlier the better. If stenosed vessels are associated with the occurrence of strokes, why wait for the stroke to occur in the first place? This is the rationale behind an approach to operate even in asymptomatic patients.

On the other hand, carotid endarterectomies (CEA) are not free of complications, the frequency of which may differ from center to center and from surgeon to surgeon. Currently the diagnosis of carotid stenosis is made by reliable non-invasive tools like Doppler ultrasound, CTangiography (CTA) or MR-angiography (MRA), which provide an accurate diagnosis of carotid stenosis. The most widely used tools are Doppler ultrasound and CTA. One of the advantages of these methods is their good inter-observer agreement. Only very rarely are the results of imaging technically poor, requiring resort to old conventional direct intra-arterial angiography.

Randomized controlled trials evaluating the role of CEA, conducted in the 1990s revealed inconclusive and controversial results as to the usefulness of CEA. Recently carotid stenting became a new attractive "bloodless" option. Unfortunately, the comparison between CEA and carotid stenting resulted in conflicting conclusions.
Unlike symptomatic carotid stenosis, ACAS carries a low risk for ipsilateral stroke and in the current "aggressive" therapeutic approach with statins and hypertension lowering drugs, the risk of strokes is even smaller. Therefore, the number needed to treat by CEA increases.

However, it is obvious that there is a sub-group of ACAS patients with higher risk of stroke than average, in whom surgery might be justified. An attempt to identify this subgroup (or sub-groups) by using advanced imaging techniques like TCD and Doppler were carried out, and a current study where TCD with microemboli detection (MES) was used revealed encouraging positive results (Lancet Neurol. Published online May 28, 2010).

Taking all the above mentioned arguments in consideration it is clear that CEA might have a beneficial effect in a selected high risk sub-group of ACAS patients that should be identified by using various advanced techniques like MES, as well as MRI to identify the thickness of the fibrous cups or various inflammatory biomarkers to assess plaque activity. These novel techniques may identify the vulnerable thrombogenic atherosclerotic plaque, leading to CEA. Otherwise, aggressive medical therapy of risk factors is recommended.

Natan Bornstein and Amos Korczyn 